Draft for Phenomenology and the Cognitive Sciences: Actions and Choking special issue

Consciousness and Choking in Visually-Guided Actions

Johan M. Koedijker ${ }^{1,2} \&$ *David L. Mann ${ }^{2}$

${ }^{1)}$ Institute of Sport Science, University of Bern, Switzerland

2) Research Institute MOVE, Faculty of Human Movement Sciences, VU University Amsterdam, the Netherlands 


\begin{abstract}
Choking under pressure describes the phenomenon of people performing well below their expected standard under circumstances where optimal performance is crucial. One of the prevailing explanations for choking is that pressure increases the conscious attention to the underlying processes of the performer's task execution, thereby disrupting what would normally be a relatively automatic process. However, research on choking has focused mainly on the influence of pressure on motor performance, typically overlooking how it might alter the way that vision is controlled when performing these motor actions. In this article we ask whether the visual component of expert motor-skill execution is susceptible to choking much like the motor component is thought to be. To do so, we draw heavily on empirical findings from studies of sporting expertise, in particular focussing on the role of gaze in three types of visually-guided actions: interceptive actions, aiming tasks, and for anticipatory skill. For each of these skills we (i) evaluate the nature of the expert advantage, (ii) discuss the role of consciousness in their control, (iii) examine the potential impact of pressure on task performance, and (iv) consider interventions designed to reduce the likelihood of choking when performing these tasks.
\end{abstract}




\section{Consciousness and Choking in Visually-Guided Actions}

Choking under pressure is a metaphor often used to describe an athlete performing below what would be expected of them in instances where there are large incentives to perform (Baumeister, 1984). The dominant explanation for this phenomenon proposes that choking occurs when automatic control processes that are typically associated with a well-learned task become 'deautomatized' or break-down as attention is 'reinvested' in the routine for the motor action (Beilock \& Carr, 2001; Carr, this issue; Deikman, 1966; Masters, 1992). Research on choking has focused primarily on the influence of pressure on overt bodily motor actions, typically overlooking how pressure might change the way that vision is controlled when performing these actions (e.g., Beilock \& Carr, 2001; Hardy, Mullen \& Jones, 1996; Liao \& Masters, 2001; Masters, 1992; Mullen, Hardy \& Tattersall, 2005. The aim of this article is to examine whether the visual component of expert motor-skill execution is susceptible to choking much like the motor component is thought to be. To do so we accept the current explicit monitoring account of choking and performance under pressure (e.g., see Cappuccio \& Ildain-Agurruza, this issue; Carr, this issue; Gottlieb, this issue) and explore the implications of this theory for the role of gaze in visual-motor actions commonly performed by skilled athletes in situations characterised by elevated levels of perceived pressure to perform.

Paying attention to the execution of a motor action is thought to be detrimental to the performance of a well-learned motor action, yet beneficial when performing a relatively new one (Beilock \& Carr, 2001; Carr, this issue). A basic understanding of the classic theories of skill acquisition helps to explain why this might be the case. Typically, the early stages of learning are thought to be highly reliant on the use of declarative knowledge (consciously accessible, factual knowledge) whereas the latter stages are much less so (Anderson, 1993; 
Fitts \& Posner, 1967; Masters \& Maxwell, 2004). For instance, Fitts and Posner (1967) proposed that learning consists of three sequential stages: the cognitive, associative, and autonomous stages. During the cognitive stage movements are controlled in a conscious manner and are characterised by substantial cognitive engagement while learners pay close attention to the step-by-step execution of their actions. As a result, it is not surprising that attention should be beneficial for performance. When the learner has acquired the fundamentals of the movement, learning progresses to the associative stage, characterised by more consistent movement execution with at least some parts of the movement controlled in a relatively automatic fashion, allowing more attention to be directed towards other aspects of the task. After extensive practice the learner may reach an autonomous stage characterised by fluent, effortless movements that require little or no attention. As the learner becomes more skilful, movement execution becomes less reliant on declarative knowledge, and instead is said to be reliant on procedural knowledge (non-conscious comprehension of how to do perform a task). This in many ways is akin to Dreyfus's (2002) concept of absorbed coping. As a result, it should not be so surprising that performance is seen to decrease when skilled athletes pay attention to their well-learned motor actions.

The idea that skill acquisition progresses from a declarative to a procedural type of control brings about the hypothesis that the motor behaviour of skilled individuals who have reached the latter stage of learning may, under specific circumstances, 'regress' to an earlier stage of learning (e.g., under heightened stress; see the progression-regression hypothesis, Fuchs, 1962). Evidence for this 'regression' hypothesis has been provided by findings of novice-like behaviour in experts under stressful conditions (e.g., Deschamps, Nourrit, Caillou, \& Delignières, 2004), and particularly by the consistent finding that the performance of experts declines under conditions that prompt attention towards the step-by-step control of 
movement execution (e.g., Beilock, et al., 2002; Gray, 2004; Koedijker, et al., 2011). For instance, Beilock, Wierenga, and Carr (2002) found that experts increased their preparation time before initiating their putt when using a strangely shaped and weighted 'funny putter', implying that they increased the amount of attention devoted to their movements. This is said to be evidence that expert motor performance can be disrupted by "re-instantiation of explicit skill-monitoring and control mechanisms" (Beilock et al., 2004, p. 379), a phenomenon described by Masters (1992) as 'reinvestment'. These findings provide a potential explanation for the reason that athletes choke under pressure: reinvestment can occur under circumstances that encourage attention to be allocated towards the step-by-step control of movement execution. Presently the dominant explanation for choking proposes that pressure is one of these circumstances, resulting in a disruption to the automatic processes of control.

In this article we wish to ask whether the visual component of expert motor-skill execution suffers the same disruption to automatic control experienced by motor skills. Research on choking has so far focused primarily on the influence of pressure on motor performance (e.g., Beilock \& Carr, 2001; Hardy, et al., 1996; Liao \& Masters, 2001; Masters, 1992; Mullen, et al., 2005), typically overlooking the role of vision in controlling these motor actions. Yet vision and action are inextricably linked in a reciprocal relationship (Bootsma \& van Wieringen, 1990; Gibson, 1979). Most emperical studies of performance under pressure examine performance in tasks that can be classed as being visually-guided actions, that is, actions that rely to a large extent on the use of visual information to guide and control the motor system to successfully perform the action (e.g., golf putting, Beliock \& Carr, 2001; soccer dribbling, Beilock et al, 2002; or baseball batting, Gray, 2004). As a result, it is surprising that more has not been done to understand if and how the visual system might respond to pressure. 
A characteristic feature of visually-guided actions is that vision can be used for the continuous guidance of the movement as it unfolds, rather than it being a pre-programmed immutable movement that does not change once it has commenced (Bootsma \& van Wieringen, 1990; Gibson, 1979). A particularly useful way to help understand how visual information is used in visually-guided actions is through the measurement of visual gaze behaviour. Visual gaze refers to where the eyes are directed when performing a task; the eyes generally move so that central vision can be directed towards the most useful information for successful task execution. Expert athletes are known to rely on gaze behaviour that is distinct from that relied on by lesser-skilled performers and this expert advantage helps to underpin their superiority in their specific sporting domain. We will look at the role of gaze in three specific types of action associated with visually-guided movements: (1) interceptive actions where a performer acts to make contact with a moving target (e.g., when hitting a baseball or catching a ball); (2) aiming tasks where an actor attempts to direct an object towards a stationary target (e.g., hitting a golf ball or performing a basketball free-throw); and (3) anticipatory actions that allows skilled performers to predict the outcome of an opponent's movement (and afford more time for the visually-guided action) based on that opponent's movement pattern. Each of these three types of skill constitutes a well-researched feature of visual-motor expertise and therefore provides an excellent starting basis from which to examine the likely influence of pressure on the visual component of expert skill execution. For each of these three skills we (i) evaluate the nature of the expert advantage in the task, (ii) consider the role of consciousness in their execution, (iii) explore the potential influence of pressure on the performance of the tasks, and (iv) conclude by discussing interventions designed to reduce the likelihood of choking when performing each task.

\section{Visual-Motor Expertise}




\section{Gaze in the Control of Interceptive Actions.}

Differences in gaze behaviour between skilled and lesser-skilled performers are evident for interceptive actions where an instrument is manipulated to make contact with a fast-moving object, for example when hitting a table-tennis ball, cricket ball, or baseball. For instance, Land and McLeod (2000) examined the gaze of cricket batters of different skill levels and found a systematic relationship between gaze and expertise. As the ball commenced its flight-path, batters attempted to track the ball with their central vision before making an anticipatory saccade (a jump eye movement) to where they expected the ball would bounce. Crucially, Land and McLeod found that batters of higher skill levels made earlier predictive saccades, suggesting a superior ability to predict the future location of the ball. More recently, Mann, Spratford and Abernethy (2013) have shown that elite cricket batters more consistently couple the rotation of their head to the flight path of the ball, and through the use of predictive eye movements are better able to co-locate their central gaze with the position of the ball at the moment of bat-ball contact. These studies provide just two brief examples of skilled performers relying on modifications to their visual gaze behaviour to underpin their skill in fast interceptive actions.

\section{Gaze in the Control of Aiming Tasks}

Visual gaze also has an important role to play in aiming tasks where the target being aimed at is static. This is best exemplified by the phenomenon of quiet eye (QE), where skilled when compared to less-skilled performers are typically found to fixate their gaze on the target for a longer period of time before executing their final critical movement (Vickers, 1996). For instance, skilled basketball players performing free-throw shots are found to spend more time than less-skilled players focussing on the ring before extending their elbow to release the ball (Vickers, 1996). This QE period has not only been shown to be longer as the 
skill level of the performer increases, but in some cases it is also found to be longer for successful outcomes than it is for unsuccessful outcomes (e.g., successful vs. unsuccessful free-throws; Mann et al., 2007). Although the neural and functional mechanisms behind the QE are still largely unclear, it is widely recognised and examined characteristic of sporting expertise.

\section{Gaze in Visual Anticipation.}

Visual expertise is not only visible whilst intercepting a target, but also commonly in the lead-up to the task when attempting to anticipate the direction of the target. Visual anticipation is the ability to quickly and accurately predict the outcome of an opponents' action as early as possible (Abernethy \& Russell, 1987; Jones \& Miles, 1978). For instance, studies in anticipation demonstrate that expert soccer goalkeepers can more accurately predict the direction of a penalty kick at an earlier point in time than a less-skilled player can (e.g., at the moment of foot-ball contact), allowing the expert keeper more time to produce the most appropriate response. Skilled athletes use the bodily cues of their opponent to anticipate the outcomes of that action as early as possible. These anticipatory judgments are often underpinned by task-specific adaptations in gaze behaviour. For example, Savelsbergh et al. (2002) showed that elite soccer goalkeepers used search strategies that relied on fewer fixations of longer duration to more 'information-rich' areas such as the kicking leg, non-kicking leg, and ball, whereas the non-elite goalkeepers fixated more on the trunk, arms, and hips of the kicker. In particular, Savelsbergh et al. (2005) found that expert goalkeepers who were more successful in simulated penalty kick situations spent longer periods of time fixating on the non-kicking leg of the kicker than the less successful goalkeepers did. Collectively the results show that while the optimal pattern of gaze is very specific to the anticipatory task being performed, in general there are differences in the way that expert 
performers search for information to underpin their superior anticipatory skill.

\section{Conscious Awareness of how to Perform Visually-Guided Actions}

When compared to the philosophical literature, in sport science there has only been a rather trivial consideration of the boundaries of consciousness. This is surprising considering the debates around the "proceduralisation" or "automatisation" of the well-learned actions of elite athletes. Here we consider the conscious control of action to constitute awareness of the specific components of an action as a scaffold to support control during action execution. Further, we make a distinction between conscious and unconscious awareness (e.g., see Shepherd, 2014). Conscious awareness of action refers to the ability to report, reconstruct or reflect on the specific components of an action. Unconscious awareness would then constitute an inability to report the defining principles of an action. A common example of unconscious awareness would be the phenomenon of blindsight (Weiskrantz, 1986), where people with specific brain lesions are able to adequately navigate past obstacles without being able to describe where they are located. Similarly, the use of subliminal feedback can provide subconscious awareness of an outcome, and can even be used as a means of providing subconscious feedback to enhance motor learning whilst minimising the amount of declarative knowledge about how to perform that action (Masters, Maxwell, \& Eves, 2009).

The explicit monitoring explanation for choking suggests that pressure results in a regression from automatised (sub-conscious) to conscious movement control (Beilock \& Carr, 2001; Deikman, 1966; Masters, 1992). If one accepts this then the question of whether visual skills choke requires an examination of how athletes might control the execution of their visually-guided actions. To do this we ask two specific questions. First, we seek to discover to what degree skilled athletes might be consciously aware of how they perform their visually-guided actions. Second, we ask to what degree this level of conscious awareness 
might change as proficiency in a skill develops.

\section{Conscious Awareness of Gaze in the Control of Visually-Guided Actions.}

Surprisingly, very little work has been done to examine whether expert performers are aware of the visual strategies they use when guiding their motor actions towards targets that are or are not moving. As a result we can only look towards case-studies and/or anecdotal accounts from our own work that examines the gaze of skilled motor performers. For instance, Figure 1 shows where a skilled junior soccer goalkeeper looks relative to a ball kicked in the air towards him. The figure shows that, rather than using his central vision to consistently track the ball, the skilled goalkeeper uses a series of jump eye movements to 'catch-up' after his gaze has fallen behind the path of the ball. This pattern of gaze was seen for most of the goalkeepers tested, and while it is not yet clear why they might rely on this saccadic strategy (similarly at least one player was found to produce these saccades ahead of the ball), it is clear that the players are not consciously aware of this saccadic strategy. When asked to indicate where they thought they were looking when presented with a series of images showing the flight-path of the ball coming towards them, the same player reported that his gaze was always directed towards the ball and that he could not recollect any times at which his gaze was not aligned with the ball. Similarly in a recent examination of the gaze behaviour of some of the world's best cricket batters, Mann et al. (2013) found that the batters tended to move their gaze ahead of the ball towards the point where they anticipated their bat would make contact with the ball. Effectively this ensured that the batters could 'watch the ball onto the bat' when they made contact with the ball. Interestingly though, when speaking to the elite batters, their conscious accounts of being able to see the ball at contact were inconsistent. For two elite batters who were both found to watch the ball at the moment they hit it, one player was adamant that he does see the ball at the moment he hits it, while the 
other said that he feels that he does not, and that it is probably impossible to do so.

Please insert Figure 1 about here

In one further example, Koedijker et al. (2013) recently examined the gaze of experienced mountain bikers navigating at high speed around a tight corner. In a debrief conducted after the experiment, participants were asked to recall where they thought they were looking when navigating the corner. Most could not provide any episodic recollection of their gaze behaviour, though a few did provide detailed accounts of where they thought they were looking. For instance, one highly-skilled participant claimed that he only changed his gaze once, reporting that he looked at the outside of the entrance of the curve and then switched as soon as possible to the inside of the exit of the curve, in essence making only one gaze shift. Surprisingly, this particular participant actually shifted his gaze on average five times during his navigation around the corner. Collectively these studies highlight how un-aware elite athletes often are of the gaze behaviours they rely on when performing motor actions, or that their conscious perception of what they 'see' is not always aligned with the location of their central vision and the movement of their eyes.

\section{Conscious Awareness of Gaze in Visual Anticipation.}

Studies of anticipatory skill provide further evidence that visually-guided tasks can often be performed in a sub-conscious manner. For instance, Mann, Abernethy and Farrow (2010) asked cricket batters to anticipate the direction of balls bowled towards them when their vision was occluded (using liquid crystal occlusion goggles) at the moment of ball release. The skilled batters were able to anticipate the direction of the ball at better-than-chance levels when they attempted to move in the direction of the ball, yet their 
performance was no better than that achievable by chance when they verbally anticipated the direction. This result suggests that anticipatory skill is embodied in the batters movements to a degree that is not apparent or consciously accessible when making verbal judgements. Dicks, Button and Davids (2010) measured the gaze behaviour of skilled football goalkeepers who similarly attempted to anticipate the direction of penalty kicks when performing movements or verbal judgements. They found that the goalkeepers directed their gaze towards different locations when performing the verbal task when compared to those for the movement task. This result is consistent with the idea that the goalkeepers rely on different information when asked to perform a less familiar verbal judgement and may be a consequence of their lack of awareness of the information they attend to when performing the natural movement task.

Collectively these examples suggest that skilled performers have very little conscious awareness of the visual strategies they use when performing visually-guided tasks. It is not our intention to suggest that skilled motor actions are performed in a vacuous state where there is no conscious thought whatsoever (see Shepherd, 2014); rather, the inference here is that skilled performers lack the conscious knowledge of how they perform visually-guided skills, much like they appear to do for well-learned motor actions. What is even less clear though is whether visual skills initially need to go though a 'declarative' stage of learning to reach this more automated state much like motor actions are thought to do. We now turn to a brief consideration of this issue.

\section{Stages of learning in visual skill.}

It is rather surprising that, to-date, there has been very little empirical work done to uncover how gaze strategies might develop as a result of learning. One exception is a study by Sailer, Flanagan, and Johansson (2005) who had participants learn to control a computer cursor with the aim to intercept targets shown on a screen as quickly as possible. Sailer et al. 
found that anticipatory gaze strategies developed as a result of learning. When first learning the task the participants typically followed the cursor with their central vision, but with practice gaze started to systematically lead (i.e., be ahead of) the movements. As eye movements change from being reactive to proactive as a result of learning, it seems likely that different information will be extracted during the different stages of learning (see also Wolpert, Diedrichsen, \& Flanagan, 2011), and/or at least that different attentional mechanisms are employed in those different stages of learning. Similar to the view that motor learning progresses in stages from a very procedural (conscious) early stage through to an automatized (subconscious) skilled stage, the visual system might also progress through different stages of learning, leading to the possibility that visual skill might also fall back to an earlier stage as a result of reinvestment under pressure. However, at this point we possess only a rudimentary understanding of how visual skill, and in particular how gaze develops as a result of learning. Clearly there is a need for the development of a theory of learning for patterns of visual gaze to provide a sound framework from which to explore how attention and performance pressure might influence visually-guided actions.

\section{Visual Skill under Pressure}

Only in the last few years has the idea that anxiety and/or pressure could influence visual gaze started to attract empirical investigation in the fields of expertise and skill acquisition. But the early evidence suggests that anxiety does alter where people look and for how long they look there. For example, Nieuwenhuys et al. (2008) asked novice rock climbers to perform identical traverses on a climbing wall in low- and high-anxiety conditions (performed 0.44 and $4.25 \mathrm{~m}$ above the ground respectively). Movement times to perform different grips increased when participants were higher on the climbing wall. Moreover, the gaze behaviour of the climbers changed when high on the wall: fixations were longer in 
duration, and climbers relied on a greater number of exploratory fixations towards the grips. Nieuwenhuys and Oudejans (2010) also found a shift in the visual search strategy of police officers if pressure was applied when performing a shooting simulation drill. Police officers took part in a shooting exercise in a low-anxiety condition against a non-threatening dummy simulating a police officer, and a high-anxiety condition against an actual opponent who regularly shot back using soap cartridges that cause pain upon impact. Targets were placed on the chest and legs of the opponent (or dummy). Results showed that with high anxiety participants generated faster but less accurate responses. Moreover, an analysis of the gaze behaviour showed that participants in the high-anxiety condition spent less time looking towards the targets and more time towards the gun and head of the opponent (see also Wilson, Wood, and Vine, 2009; Causer, Holmes, Smith \& Williams, 2011). Collectively across these studies it has been proposed that higher anxiety results in gaze being distracted from attending to the information most crucial for task performance. Instead, these studies suggest that anxiety leads to gaze being directed towards the features of the environment that might be essential for survival (i.e., an opponent's gun, or grips on a climbing wall) rather than the information which might be most informative or efficient for the primary task being performed. These results do provide some evidence to show that pressure could influence gaze in a similar fashion to the way that it does motor skill.

The majority of research on the relationship between increased pressure and visual skill in sporting expertise has so far relied on QE as a marker of visual expertise (e.g. Behan \& Wilson, 2008; Wilson, Vine, \& Wood, 2009). For instance, Wilson et al. (2009) examined the influence of anxiety on basketball shootng by asking basketball players to take free-throw shots in high-anxiety and in control conditions. In the high-anxiety condition, participants were informed that their performance was to be compared to that of their teammates and other 
teams within the same competitive league, and that there would be financial rewards for the best performers (see Carr, this issue, for an excellent discussion of these types of pressure manipulations). In the control condition, participants were instructed to do their best, but that their performance would not to be used for comparison with other participants. Wilson et al. found that the QE period, that is, the final fixation on the ring before the final extension of the arms, was reduced by $34 \%$ in the high-anxiety condition. Further, more fixations of shorter duration were made in the high-anxiety condition and more of those fixations were made towards targets in the vicinity of the hoop rather than towards the hoop itself (see also Behan \& Wilson, 2008), demonstrating a shift away from expert visual search behaviour when under pressure. Over the past five years a range of studies exploring the association between pressure and QE have collectively supported the view that pressure has a significant impact on the QE period (e.g., Moore et al., 2012; Wilson, et al., 2009). Anxiety, resulting from competitive pressure, generally results in a shortening of the QE, particularly the proportion occurring after the critical movement has been initiated. These results suggest that performance failure under pressure might be closely related to disruptions in the visual control of action.

\section{Do Visual Skills ‘Choke’ under Pressure?}

It appears that visual search behaviour changes when the performer is placed under pressure, typically reflected by shorter QE durations (e.g. Behan \& Wilson, 2008; Wilson, et al, 2009), increases in the number of fixations (Nieuwenhuys et al., 2008) and more fixations on locations less relevant for successful task performance (e.g. Nieuwenhuys \& Oudejans, 2010; Nieuwenhuys et al., 2008). These results could be interpreted as evidence that visual gaze is susceptible to choking much like motor actions are. However, the absence of a suitable theoretical framework on how visual search strategies change over the course of skill 
acquisition makes it difficult to distinguish whether changes are the result of a regression towards the type of control relied on when learning commenced (as is said to be the case for choking), or whether there are other potential explanations for the changes, for instance functional shifts towards sources of information deemed more essential for 'survival' or to account for the threatening state being experienced.

The interest shown in the effect of pressure on QE is yet to be shown in other types of visually-guided tasks. Particularly notable are the lack of empirical studies investigating the effect of pressure on gaze in interceptive tasks (e.g. cricket and baseball batting) and for anticipatory skill. The lack of a suitable theoretical framework on how visual search strategies change over the course of skill acquisition means that we can only speculate how pressure might influence gaze in these tasks. Following the limited research that shows pressure causes gaze to adopt more novice-like strategies, one might expect pressure to reduce (or at least delay) the number of predictive eye movements used by experts when performing interceptive actions. Alternately, if skilled athletes are not aware that they use these predictive gaze strategies then - based on the theory of explicit monitoring - they may be unable to revert to a form of novice-like strategy if they possess no explicit awareness of their previously learned strategies. Clearly this is a potentially fruitful area for future research. Similarly, very little is known about how pressure might influence anticipatory skill (though see Alder et al, 2014 for a recent exception). Along similar lines it might be expected that pressure might result in a reduction in the number of fixations allocated towards more 'sophisticated' body locations whose movements correlate strongly with the likely action outcome (e.g., the non-kicking leg of a penalty kick taker), with a concurrent increase in the number of fixations towards the less informative cues (e.g., the trunk, arms and hips). Again, this appears to be a question worthy of investigation. 


\section{Preventing choking?}

If vision is as affected by pressure as early evidence appears to suggest it is then this opens the possibility for visual-motor skills to be trained in a way that decreases the likelihood of skill failure under pressure. A number of training interventions have been designed in an effort to teach motor skills in a manner that decreases the likelihood that the skill will 'break-down' under pressure (e.g. Liao \& Masters, 2001; Masters, 1992; Maxwell et al., 2001). Most commonly these learning strategies come under the banner of implicit learning, where motor skill is acquired whilst trying to minimise the acquisition of explicit (declarative) knowledge about how to perform the skill. This is done to reduce the likelihood that performers will revert to the use of declarative knowledge when placed under pressure. However to-date, these interventions have primarily been aimed at improving the resilience of motor rather than visual skills. For instance, Masters (1992) found that secondary cognitive tasks could be used to divert attention away from movement control during learning to increase the automaticity of motor control. As a consequence, learners acquired less explicit knowledge about how to perform the specific skill. In this study, a 'dual-task' approach was used to distract the attention of novice golfers who learned to putt golf balls whilst randomly generating and calling out letters of the alphabet. The dual-task learners were found to have accrued little knowledge about what movements and techniques they were using when performing the golf-putting task. This suggests that diverting attention during learning circumvented the generation of conscious, declarative memory processes and encouraged the development of automatic, procedural knowledge early in motor-skill learning. Importantly, the performance of these dual-task learners was unaffected under conditions evoking pressure (financial reward and evaluation of an expert), whereas the performance of participants who learned without the secondary task decreased when placed under pressure. 
The idea that implicitly learned skills are more robust to psychological pressure has been repeatedly demonstrated using a range of different approaches (e.g., analogy learning, Liao \& Masters, 2001; errorless learning, Maxwell, et al., 2001; and subliminal feedback, Masters, et al., 2009). The underlying philosophy across each of these practice designs is that bypassing the declarative stage of learning can decrease the likelihood that stored explicit rules are activated in working memory when under pressure (Masters \& Maxwell, 2004). Given the possible parallels between motor- and visual-skill learning, implicit learning may provide a beneficial means of training visual-motor and anticipatory skill in an effort to increase robustness to performance pressure (e.g. Farrow \& Abernethy, 2002; Williams, Ward, Knowles \& Smeeton, 2002).

\section{Preventing Choking in Visually-Guided Actions.}

Implicit learning strategies aimed at circumventing declarative processes during skill acquisition appear to provide a successful pathway to robust motor performance under pressure. However, learning designs used to improve the resistance of visual skills to choking do not necessarily need to be implicit to be successful. For instance, a number of studies designed to improve the QE of performers have used very explicit instructions yet still seem to lead to improvements in performance (e.g. Harle and Vickers, 2001; Vine \& Wilson, 2010; Vine \& Wilson, 2011). For instance, Harle and Vickers (2001) examined the effect of a QE training regime designed to improve the free-throw accuracy of near-elite basketball players. During the QE training program athletes received feedback about how their gaze behaviour compared to that of an expert player. Results showed that the QE-trained players significantly increased their QE durations by 300-900 ms, and this was associated with an increase in their free-throw percentages in a laboratory test and during competition. Further research has supported the positive effects of QE training for both novice and skilled performers over a 
variety of tasks including in golf putting (Vine, Moore, \& Wilson, 2011), in simulated laparoscopic surgery (Vine \& Wilson, 2011), and in rifle and shotgun shooting (Causer, Holmes, \& Williams, 2011) .

The positive effects of QE training in conjunction with the link between QE and performance under pressure has spurred a number of intervention studies designed to establish whether QE training improves performance under pressure. Vine and Wilson (2010) used a QE training design that consisted of five explicit instructions rephrased into QE beneficial terminology for golf putting (e.g. "Your final fixation should be on the back of the ball and for no longer than 2-3 seconds"), while participants in a control group received five similar technical instructions (e.g. "Keep the putter head square to the ball"). Results revealed that QE-trained novice golfers had longer QE durations, as well as more accurate putting performance after practice. Furthermore, in a pressure test the QE-trained group maintained their longer QE durations and performed better than the control group. These results support the idea that QE training allowed performers to negate the adverse effects of anxiety on performance by maintaining effective visual control when under pressure (see also Vine \& Wilson, 2011).

Explicit instructions about how to direct visual gaze seem to provide an effective tool not only for elongating the duration of the QE period, but also for directing gaze in other visually-guided actions. In a task simulating laparoscopic surgery, Wilson et al. (2011) assigned novice participants to one of a gaze training, movement training, or discovery learning intervention. The gaze-training group were shown a video of where an expert looks when performing the task (shown by a cursor in footage from a gaze-tracker). Participants were made aware of the gaze cursor and were instructed to adopt the gaze strategy used by the expert. The movement-training group were shown the same video but without the gaze cursor 
present. Instead, participants were instructed to mimic the actual movements of the expert. The discovery-learning group was not provided with the video or any training instructions. However, they were allowed to examine their performance after every trial. Wilson et al. showed that participants provided with gaze information during training performed more accurately on the laproscopic task than did participants in the movement-guided or discovery learning groups. This suggests that gaze training can also improve performance of more complex visually-guided actions. Further, the performance advantage of the gaze-trained group was even more pronounced under dual-task conditions, similar to performance advantages found for implicit learning strategies. As a central characteristic of implicitly learned motor skills is robust performance under pressure, implicit gaze training might also alleviate the potentially damaging effects of pressure on gaze control.

Although many of the interventions designed to alter gaze rely on the use of very explicit instructions, it has been proposed that this training may still constitute a form of implicit learning. The methods used in these interventions encourage the performer to attend to an external target (i.e., the direction of gaze) rather than the movement pattern being produced. In support, many of the benefits of gaze training strongly resemble those arising from implicit learning, viz. robustness to increased pressure and dual-task conditions. Vine et al. (2013) investigated whether QE training could constitute a form of implicit learning. Forty-five novice participants practiced golf putts in one of three training groups: (i) an explicit-learning group who received six explicit instructions related to the mechanics of their putting stroke (see also Vine \& Wilson, 2010); (ii) a QE-learning group who viewed video footage showing the gaze behavior of an elite performer in addition to receiving the six instructions received by the explicit-learning group; and (iii) an analogy-learning group who were asked to model their putt using the analogy of a grandfather clock, that is, participants 
were instructed to "keep your body still like a grandfather clock and use your arms the same way that the pendulum of the clock operates". The results showed not surprisingly that the QE-trained group had longer periods of QE than both other groups, but that the QE- and analogy-groups both reported less explicit rules for how they performed their golf putts than the explicit-learning group did. Importantly, the QE-group outperformed both other groups under conditions of increased pressure, indicating that QE training appears to offer advantages in terms of robustness to pressure and expedited skill acquisition, similar to other established implicit learning techniques.

\section{Preventing choking in visual anticipation.}

Many of the early studies designed to train anticipatory skill relied on the use of explicit instructions about the most informative or 'information rich' areas towards which observers should focus to provide the most appropriate anticipatory responses (e.g., Abernethy, Wood \& Parks, 1999; Farrow, Chivers, Hardingham, \& Sasche, 1998; Williams, Ward, Knowles \& Smeeton, 2002). However, in response to the potential benefits of implicit motor learning, similar implicit approaches have been adopted in an effort to improve the way that anticipatory skills are learned. For instance, Farrow and Abernethy (2002) studied the efficacy of an implicit form of learning to enhance the anticipation of the direction of tennis serves. Participants in an explicit-learning group were informed about the relationship between certain movement features (e.g., the ball toss, angle of racquet head, and shoulder rotation) and the direction of tennis serves shown in video clips during training. In contrast, an implicit-learning group watched the same video clips and were asked to simply estimate the speed of each serve. This approach was adopted to direct the learners' attention towards a task-irrelevant cue, forcing unconscious implicit processes to 'pick-up' on the relationship between movement cues and serve direction. Intriguingly, the implicit-learning group 
significantly increased their anticipatory skill whereas the explicit-learning (and control and placebo groups) did not.

Hagemann et al. (2006) adopted a learning design that attempted to more directly improve the gaze behaviour of participants who were learning to anticipate the direction of badminton strokes. In their study, participants viewed video footage of badminton strokes but in a critical training group a transparent red patch was digitally superimposed onto the video footage to orient attention toward the key display features known to be relied on by skilled badminton players (viz. the position of the trunk, then arm, the racket, and finally to the point of contact). Results showed that participants who trained with cueing significantly improved their anticipatory skill better than control participants who did not receive the cueing information. Savelsbergh et al. (2010) used a similar cueing technique to enhance the anticipatory skill of novice soccer players learning to anticipate the direction of penalty kicks. They found that participants who viewed the cueing information adopted more 'expert-like' visual search patterns that led to enhanced anticipatory performance (see also Ryu, Kim, Abernethy, Mann, 2012).

Collectively these studies show that the implicit guidance of visual attention can be an effective method of improving anticipatory skill. But they don't necessarily show that performance might be resilient to induced pressure. Smeeton, et al. (2005) did so by examining anticipatory performance under pressure. Novices participants learned to anticipate the direction of tennis serves using a video-based judgement task and were assigned to one of three groups: (i) an explicit-instruction group who received ten explicit instructions about the relationship between a postural cue and its effect on shot direction (e.g. "Look at the hips and shoulders during the shot, see how they don't rotate as much as a crosscourt shot."); (ii) a guided-discovery group who also received ten instructions, but they only guided attention 
towards the relevant information location (e.g. "Look at the hips and shoulders during the shot"); or (iii) a discovery-learning group who were not given any instructions about the key postural cues, but were instead encouraged to discover the relationship between cue and shot direction with statements such as "Can you work out where she is going to hit the ball before she hits it?". When compared to the performance of a control group, all three instructional methods resulted in enhanced anticipatory skill, that is, more accurate responses and faster response times. However, when under stress, the explicit group performed significantly worse than the other two groups (see also Abernethy et al., 2012). Furthermore, the guided-discovery method resulted in more rapid initial improvements in performance than discovery learning. In sum, these studies suggest that the guided discovery method that relies on implicit learning would be the best way to train visual anticipation. Visual-motor and anticipatory skill training interventions that are based on implicit learning techniques show a similar resilience to performance pressure that is seen with implicit motor learning techniques. Not only does this provide a potential tool for practitioners to prepare athletes for the stress of competition, it also allows us to draw further parallels between motor and visual skills with respect to their development and control.

\section{Conclusions}

Here we have sought to evaluate the role of conscious and subconscious processing when controlling visually-guided actions. It is commonly understood that expert performance in motor tasks relies in large part on automatic control processes (e.g. Fitts and Posner, 1967) and here we have shown that the available empirical evidence converges towards the view that skilled athletes may rely on similarly automatic control of their visually-guided skills. The automatic nature of expert visual skill execution raises the possibility that, when put under pressure, visual skills might also 'choke'. A regression or reinvestment account of 
choking though relies on a commensurate change in the conscious awareness of how an action (or visual skill) is performed as proficiency in that skill develops. While it is possible that visual skills might regress towards a more 'novice-like' mode of control when put under pressure, the lack of a clear theory for how visual skills develop as a result of learning make it difficult to draw such a conclusion. Clearly there is a need for both an empirical and philosophical account for how visual control might change as a result of learning (e.g., see Sailer et al., 2005). So for the moment, the question of whether visual skills fall back to the type of control that was present early in learning when put under pressure remains largely unanswered. Nonetheless, interventions designed to enhance the visual control of action and anticipation appear to be surprisingly effective in increasing the robustness of these skills under pressure.

\section{Acknowledgements}

Figure 1 presents results from a Masters project performed by Rick Dumoulin and Michiel Vervoort at VU University Amsterdam with the support of Vosse de Boode and Ajax Football Club.

\section{References}

Abernethy, B., \& Russell, D. G. (1987). The relationship between expertise and visual search strategy in a racquet sport. Human movement science, 6, 283-319.

Abernethy, B., Schorer, J., Jackson, R. C., \& Hagemann, N. (2012). Perceptual training methods compared: The relative efficacy of different approaches to enhancing sport-specific anticipation. Journal of Experimental Psychology: Applied, 18, 143-153.

Abernethy, B., Wood, J. M., \& Parks, S. (1999). Can the anticipatory skills of experts be learned by novices? Research Quarterly for Exercise and Sport, 70, 313-318.

Alder, D.B., Ford, P.R., Causer, J., Williams, A.M. (2014). Perceptual training with anxiety: 
effects on the anticipatory judgements of elite athletes. Meeting of the Expertise and Skill Acquisition Network, Sheffield UK.

Anderson, J. R. (1993). Rules of mind. Hillsdale, NJ: Lawrence Erlbaum Associates.

Baumeister, R. F. (1984). Choking under pressure: self-consciousness and paradoxical effects of incentives on skillful performance. Journal of personality and social psychology, 46, 610-620.

Behan, M., \& Wilson, M. (2008). State anxiety and visual attention: The role of the QE period in aiming to a far target. Journal of Sports Sciences, 26, 207-215.

Beilock, S. L., Bertenthal, B. I., McCoy, A. M., \& Carr, T. H. (2004). Haste does not always make waste: Expertise, direction of attention, and speed versus accuracy in performing sensorimotor skills. Psychonomic Bulletin and Review, 11, 373-379

Beilock, S. L., \& Carr, T. H. (2001). On the fragility of skilled performance: What governs choking under pressure? Journal of Experimental Psychology: General, 130, 701-725.

Beilock, S. L., Carr, T. H., MacMahon, C., \& Starkes, J. L. (2002). When paying attention becomes counterproductive: Impact of divided versus skill-focused attention on novice and experienced performance of sensorimotor skills. Journal of Experimental Psychology: Applied, 8, 6-16.

Beilock, S., Wierenga, S. A., \& Carr, T. H. (2002). Expertise, attention, and memory in sensorimotor skill execution: Impact of novel task constraints on dual-task performance and episodic memory. The Quarterly Journal of Experimental Psychology, 55A, $1211-1240$

Bootsma, R. J., \& van Wieringen, P. C. W. (1990). Timing an attacking forehand drive in table tennis. Journal of Experimental Psychology: Human Perception and Performance, $16,21-29$. 
Cappuccio, M. \& Ilundain-Agurruza, J. (this issue). Phenomenology and the Cognitive Sciences.

Carr, T. (this issue). Strengths and weaknesses of reflection as a guide to action: pressure assails performance in multiple ways. Phenomenology and the Cognitive Sciences.

Causer, J., Holmes, P.S., Smith, N.C., \& Williams, A. M. (2011). Anxiety, movement kinematics, and visual attention in elite-level performers. Emotion, 11(3), 595-602.

Causer, J., Holmes, P. S., \& Williams, A. M. (2011). Quiet eye training in a visuomotor control task. Medicine \& Science in Sports \& Exercise, 43, 1042-1049.

Deikman, A. J. (1966). De-automatization and the mystic experience. Psychiatry, 29, 324-338.

Deschamps, T., Nouritt, D., Caillou, N., \& Delignières, D. (2004). Influence of a stressing constraint on stiffness and damping functions of a ski simulator's platform motion. Journal of Sports Sciences, 22, 867-874.

Dicks, M., Davids, K., \& Button, C. (2010). Individual differences in the visual control of intercepting a penalty kick in association football. Human Movement Science, 29, 401-411.

Dreyfus, H.L. (2002). Intelligence without representation - Merleau-Ponty's critique of mental representation. Phenomenology and the Cognitive Sciences, 1, 367-383.

Farrow, D., \& Abernethy, B. (2002). Can anticipatory skills be learned through implicit video based perceptual training? Journal of Sports Sciences, 20, 471-485.

Farrow, D., Chivers, P., Hardingham, C., \& Sachse, S. (1998). The effect of video-based perceptual training on the tennis return of serve. International Journal of Sport Psychology, 23, 231-242.

Fitts, P., \& Posner, M. (1967). Human performance. Belmont, CA: Brooks-Cole. 
Fuchs, A. H. (1962). The progression-regression hypothesis in perceptual-motor skill learning. Journal of Experimental Psychology, 63, 177-182.

Gibson, J. J. (1979). The ecological approach to visual perception. Boston: Houghton Mifflin.

Gottlieb, G. (this issue). Know-how, procedural knowledge, and choking under pressure. Phenomenology and the Cognitive Sciences.

Gray, R. (2004). Attending to the execution of a complex sensorimotor skill: Expertise differences, choking, and slumps. Journal of Experimental Psychology: Applied, 10, $42-54$.

Hagemann, N., Strauss, B., \& Cañal-Bruland, R. (2006). Training perceptual skill by orienting visual attention. Journal of Sport \& Exercise Psychology, 28, 142-158.

Hardy, L., Mullen, R., \& Jones, G. (1996). Knowledge and conscious control of motor actions under stress. British Journal of Psychology, 87, 621-636.

Harle, S. K., \& Vickers, J. N. (2001). Training quiet eye improves accuracy in the basketball free throw. Sport Psychologist, 15, 289-305.

Jones, C. M., \& Miles, T. R. (1978). Use of advance cues in predicting the flight of a lawn tennis ball. Journal of human movement studies, 4, 231-235.

Koedijker, J. M., Kredel, R., Schindler, S., Hossner, E.-J. (2013). Effects of vibrations on gaze behavior in high-speed navigation. Proceedings of the EuroMov Inaugural Conference, Montpellier, France.

Koedijker, J. M., Poolton, J. M., Maxwell, J. P., Oudejans, R. R., Beek, P. J., \& Masters, R. S. W. (2011). Attention and time constraints in perceptual-motor learning and performance: Instruction, analogy, and skill level. Consciousness and cognition, 20, 245-256.

Land, M. F., \& McLeod, P. (2000). From eye movements to actions: how batsmen hit the 
ball. Nature neuroscience, 3, 1340-1345.

Liao, C. H., \& Masters, R. S. W. (2001). Analogy learning: A means to implicit motor learning. Journal of Sports Sciences, 19, 307-319.

Mann, D. L., Abernethy, B., \& Farrow, D. (2010). The resilience of natural interceptive actions to refractive blur. Human movement science, 29, 386-400.

Mann, D. L., Spratford, W., \& Abernethy, B. (2013). The head tracks and gaze predicts: how the world's best batters hit a ball. PloS one, 8, e58289.

Mann, D. T. Y., Williams, A. M., Ward, P., \& Janelle, C. M. (2007). Perceptual-cognitive expertise in sport: a meta-analysis. Journal of Sport \& Exercise Psychology, 29, 457-478.

Masters, R. S. W. (1992). Knowledge, knerves and know-how: The role of explicit versus implicit knowledge in the breakdown of a complex motor skill under pressure. British Journal of Psychology, 83, 343-358.

Masters, R. S. W., \& Maxwell, J. P. (2004). Implicit motor learning, reinvestment and movement disruption. What you don't know won't hurt you? In A. M. Williams \& N. J. Hodges (Eds.), Skill acquisition in sport: Research, theory and practice (pp. 207-228). London: Routledge.

Masters, R. S., Maxwell, J. P., \& Eves, F. F. (2009). Marginally perceptible outcome feedback, motor learning and implicit processes. Consciousness and cognition, 18, 639-645.

Masters, R. S. W., Van der Kamp, J., \& Jackson, R. C. (2007). Imperceptibly off-center goalkeepers influence penalty-kick direction in soccer. Psychological science, 18, 222-223.

Maxwell, J. P., Masters, R. S. W., Kerr, E., \& Weedon, E. (2001). The implicit benefit of 
learning without errors. The Quarterly Journal of Experimental Psychology, 54A, 1049-1068.

Moore, L., Vine, S.J., Cooke, A., Ring, C., \& Wilson, M.R. (2012). Quiet eye training expedites motor learning and aids performance under pressure: The roles of response programming and external attention. Psychophysiology, 49, 1005-1015.

Nieuwenhuys, A., \& Oudejans, R. R. (2010). Effects of anxiety on handgun shooting behavior of police officers: a pilot study. Anxiety, Stress, \& Coping, 23, 225-233.

Nieuwenhuys, A., Pijpers, J. R., Oudejans, R. R., \& Bakker, F. C. (2008). The influence of anxiety on visual attention in climbing. Journal of Sport \& Exercise Psychology, 30, 171-185.

Ryu, D., Kim, S., Abernethy, B., \& Mann, D. L. (2013). Guiding attention aids the acquisition of anticipatory skill in novice soccer goalkeepers. Research quarterly for exercise and sport, 84, 252-262.

Sailer, U., Flanagan, J. R., \& Johansson, R. S. (2005). Eye-hand coordination during learning of a novel visuomotor task. The Journal of Neuroscience, 25, 8833-8842.

Savelsbergh, G. J. P., van Gastel, P. J., \& van Kampen, P. M. (2010). Anticipation of penalty kicking direction can be improved by directing attention through perceptual learning. International Journal of Sport Psychology, 41, $24-41$.

Savelsbergh G.J.P., Williams, A.M., Van der Kamp, J., \& Ward, P. (2002). Visual search, anticipation and expertise in soccer goalkeepers. Journal of Sports Sciences, 20, 279287.

Savelsbergh G.J.P., van der Kamp, J., Williams, A.M., \& Ward, P. (2005). Anticipation and visual search behaviour in expert soccer goalkeepers. Ergonomics, 48, 1686-1697.

Shepherd, J. (2014). Conscious control over action. Mind and Language. 
Smeeton, N. J., Williams, A. M., Hodges, N. J., \& Ward, P. (2005). The relative effectiveness of various instructional approaches in developing anticipation skill.Journal of Experimental Psychology: Applied, 11, 98-110.

Vickers, J. N. (1996). Visual control when aiming at a far target. Journal of Experimental Psychology: Human Perception and Performance, 22, 342-354.

Vine, S. J., Moore, L. J., Cooke, A., Ring, C., \& Wilson, M. R. (2013). Quiet eye training: a means to implicit motor learning. International Journal of Sport Psychology, 44(4), 367-386.

Vine, S.J., Moore, L.J., \& Wilson, M.R. (2011). Quiet eye training facilitates competitive putting performance in elite golfers. Frontiers in Psychology, 2, 1-9

Vine, S. J., \& Wilson, M. R. (2010). Quiet eye training: Effects on learning and performance under pressure. Journal of Applied Sport Psychology, 22, 361-376.

Vine, S. J., \& Wilson, M. R. (2011). The influence of quiet eye training and pressure on attention and visuomotor control. Acta Psychologica, 136, 340-346.

Weiskrantz, L. (1986). Blindsight: A case study and implications. Oxford University Press.

Williams, A. M., Ward, P., Knowles, J. M., \& Smeeton, N. J. (2002). Anticipation skill in a real-world task: measurement, training, and transfer in tennis. Journal of Experimental Psychology: Applied, 8, 259-270.

Wilson, M. R., Vine, S. J., Bright, E., Masters, R. S., Defriend, D., \& McGrath, J. S. (2011). Gaze training enhances laparoscopic technical skill acquisition and multi-tasking performance: a randomized, controlled study. Surgical endoscopy, 25, 3731-3739.

Wilson, M. R., Vine, S. J., \& Wood, G. (2009). The influence of anxiety on visual attentional control in basketball free throw shooting. Journal of Sport \& Exercise Psychology, 31, 152-168. 
Wilson, M. R., Wood, G., \& Vine, S. J. (2009). Anxiety, attentional control, and performance impairment in penalty kicks. Journal of Sport \& Exercise Psychology, 31, 761-775.

Wolpert, D. M., Diedrichsen, J., \& Flanagan, J. R. (2011). Principles of sensorimotor learning. Nature Reviews Neuroscience, 12, 739-751. 


\section{Figure \& Caption}

Figure 1. Visual angles subtended by the ball and gaze for a football goalkeeper catching a ball kicked towards him from $28 \mathrm{~m}$. As the ball or gaze moves further above the ground the visual angle increases. The position of gaze relative to the ball shows that a series of catch-up eye movements are used after gaze progressively lags below/behind the ball. All measures are expressed in degrees relative to the starting position of the ball.

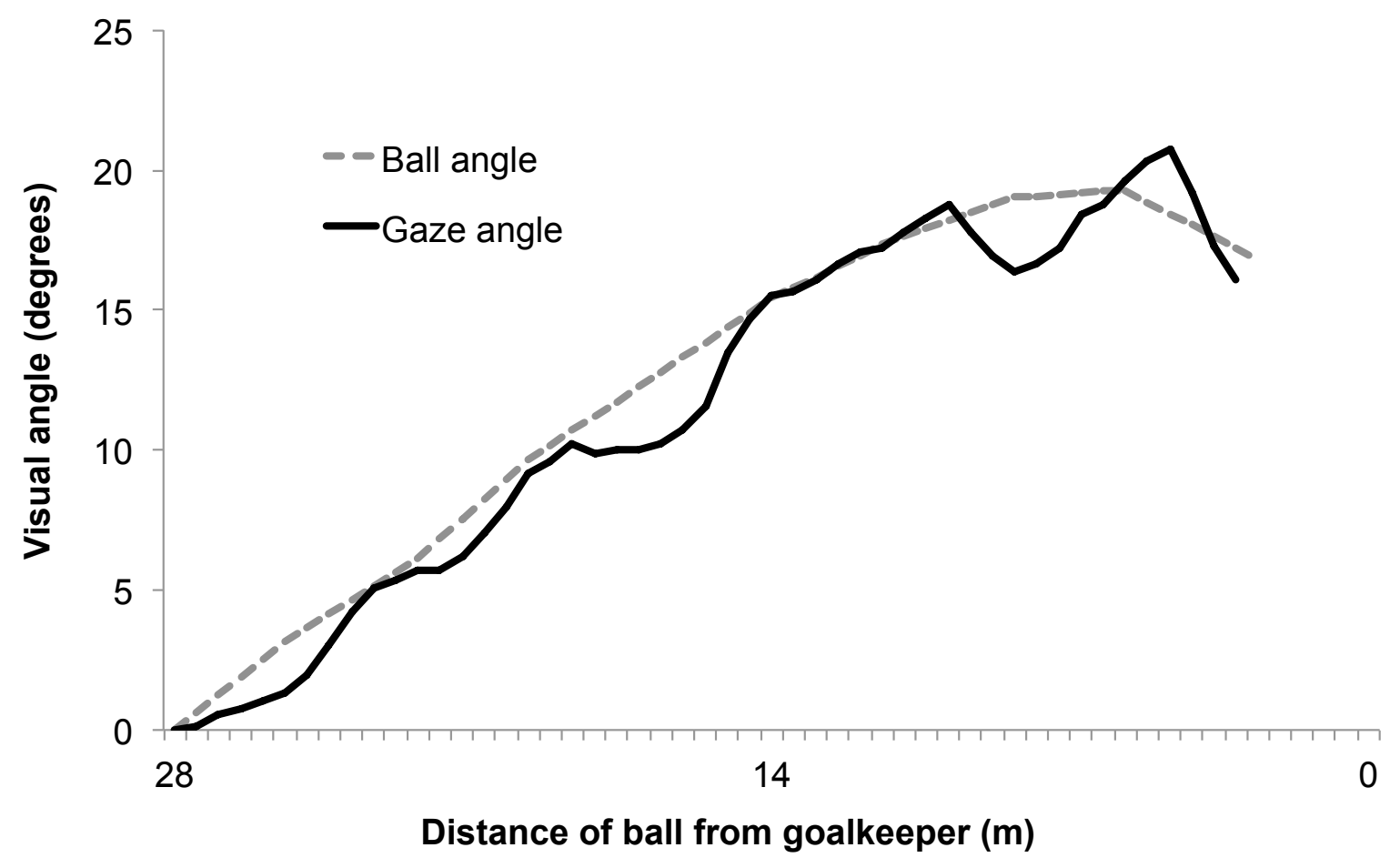

